\title{
Suppression of MD2 inhibits breast cancer in vitro and in vivo
}

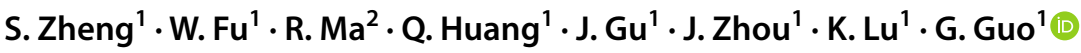

Received: 25 January 2021 / Accepted: 7 March 2021 / Published online: 17 March 2021

(c) The Author(s) 2021

\begin{abstract}
Purpose To explore the effects of the intervening measure targeting myeloid differentiation 2 (MD2) on breast cancer progression in vitro and in vivo.

Methods The expression of MD2 in normal breast cells (Hs 578Bst) and three kinds of breast carcinoma cell lines (MCF7, MDA-MB-231 s and 4T1) were detected by western blot. MTT assay was used to detect the proliferation of 4T1 cells treated by $\mathrm{L} 6 \mathrm{H} 21$, cell migration and invasion was measured by wound healing assay and trans-well matrigel invasion assay, respectively. In addition, to further study the role of MD2 in tumor progression, we assessed the effects of inhibition of MD2 on the progression of xenograft tumors in vivo.

Results The expression of MD2 is much higher in MDA-MB-231 s and 4T1cells than that in normal breast cells (Hs 578Bst) or MCF-7 cells $(p<0.05)$. In vitro, suppression of MD2 by L6H21 has a significant inhibition of proliferation, migration and invasion in 4T1 cells in dose-dependent manner. In vivo, L6H21 pretreatment significantly improved survival of 4T1-bearing mice $(p<0.05)$. Additionally, we also observed that none of the mice died from the toxic effect of $10 \mathrm{mg} \mathrm{kg}^{-1} \mathrm{~L} 6 \mathrm{H} 21$ in 60 days.

Conclusion Overall, this work indicates that suppression of MD2 shows progression inhibition in vitro and significantly prolong survival in vivo. These findings provide the potential experimental evidence for using MD2 as a therapeutic target of breast carcinoma.
\end{abstract}

Keywords Breast neoplasms $\cdot$ Myeloid differentiation $2($ MD2) $\cdot$ Proliferation $\cdot$ Migration $\cdot$ Invasion

\section{Abbreviations}

MD2 Myeloid differentiation 2

TLRs Toll like receptors

PRRs Pathogen recognition receptors

PAMPs Pathogen-related molecular patterns

DAMPs Damage-related molecular patterns

TRIF TIR domain-containing adapter-inducing interferon- $\beta$

MYD88 Myeloid differentiation primary response

L6H21 (E)-2,3-dimethoxy-4'-methoxychalcone

FBS Fetal bovine serum

S. Zheng and W. Fu equally contributed as first authors.

G. Guo

guoguilong@sina.com

1 Department of Breast Surgery, The First Affiliated Hospital of Wenzhou Medical University, Ouhai District, Wenzhou 325000, Zhejiang, China

2 Department of Breast Surgery, The Second Affiliated Hospital of Wenzhou Medical University, Lucheng District, Wenzhou 325000, Zhejiang, China
OD The optical density

LPS Lipopolysaccharide

RNAi Ribonucleic acid interference

\section{Introduction}

Breast cancer is one of the most common cancers affecting women worldwide [1]. Despite intensive efforts and remarkable advances in the management of breast cancer, the physiological conditions that lead to tumorigenesis including breast cancer are not well understood and distant metastasis are still occurred in part of patients after treatments. It was reported that over $90 \%$ of the deaths of cancer patients are caused by metastasis [2]. Therefore, finding new modalities that treat the local and systemic components of the disease has become increasingly important.

Toll like receptors (TLRs) belong to the pathogen recognition receptors (PRRs) family, which are essential components of innate immune system and serve as major contributor to chronic inflammation [3]. Through identifying 
pathogen-related molecular patterns (PAMPs) and damagerelated molecular patterns (DAMPs), TLRs plays a central role in the immune response [4]. There are two pathways that TLRs use to send their messages to regulate cell functions. TLR3 uses TIR domain-containing adapter-inducing interferon- $\beta$ (TRIF), while TLR1, TLR2, TLR5, TLR6, TLR7, TLR8 and TLR9 use myeloid differentiation primary response (MYD88). TLR4, however, uses both MYD88 and TRIF pathways for transduction of its signaling [5]. TLRs binding to ligands can activate a variety of cascade signal transduction pathways and promote the release of inflammatory mediators, such as cytokines, chemokines, and ultimately promote tumor cell growth, angiogenesis and lymphangiogenesis, tumor infiltration and metastasis $[6,7]$. As one of the most unique TLRs, TLR4 has already been linked to tumors including breast cancer and has been implicated in low overall survival rate [8-11].

MD2, well known as an accessory protein of TLR4, plays an essential role in activation of TLR4 signaling pathway in inflammatory response [12]. However, whether MD2 has a similar effect on the progression of breast cancer is still poorly understood and there was rare evidence indicating the correlation of MD2 and tumor progression. Previously, our cooperators synthesized a new chalcone derivative, (E)-2, 3-dimethoxy-4'-methoxychalcone (L6H21), which identified MD2 as its molecular target. In addition, they demonstrated that $\mathrm{L} 6 \mathrm{H} 21$ shows excellent inhibition of the TLR4-mediated inflammatory response and septic injury both in vitro and in vivo [13]. Therefore, to investigate whether L6H21 has antitumor effects, in this study, we first detected the expression of MD2 in four kinds of cell lines by western blot assay. Next, we aimed to observe and confirm that the effects of $\mathrm{L} 6 \mathrm{H} 21$ on 4T1 cells proliferation, migration and invasion in vitro. Additionally, model of transplanted tumor on $\mathrm{BALB} / \mathrm{c}$ nude mouse were used to study the anticancer effect of L6H21 in vivo.

\section{Materials and methods}

\section{Cell culture}

Normal breast cells (Hs 578Bst) and breast cancer cell lines MCF-7 (estrogen receptor-positive, HER2-negative breast cancer), MDA-MB-231 s (the estrogen receptor-negative, progesterone receptor-negative and HER2-negative human breast cancer cells) and 4T1 (spontaneously metastasizing mammary adenocarcinoma) were obtained from ATCC and grown according to ATCC recommended culture conditions. MDA-MB-231 s and 4T1 cells represent highly malignant breast cancers. The cells were cultured in DMEM (Gibco) or RPMI 1640 medium (Gibco) with 10\% fetal bovine serum
(FBS) (Gibco), and then incubated at $37^{\circ} \mathrm{C}$ in a humidified atmosphere containing $5 \% \mathrm{CO}_{2}$ and $95 \%$ air.

\section{Western blot}

Cell protein samples $(50 \mu \mathrm{g})$ were subjected to $10 \%$ SDSPAGE and transferred onto a PVDF membrane (Bio-Rad Laboratories). After being blocked in blocking buffer (5\% milk in tris-buffered saline containing $0.05 \%$ Tween 20 ) for $1.5 \mathrm{~h}$ at room temperature, membranes were incubated with different primary antibodies overnight at $4{ }^{\circ} \mathrm{C}$. The membranes then were washed in TBS-T and reacted with secondary horseradish peroxidase-conjugated antibody (Santa Cruz, CA, USA;1:5000) for $1-2 \mathrm{~h}$ at room temperature. Blots were then visualized using enhanced chemiluminescence reagents (Bio-Rad Laboratories). The density of the immunoreactive bands was analysed using Image $\mathbf{J}$ software (NIH, Bethesda, MD, USA).

\section{MTT assay}

Cell proliferation was determined using the MTT method. Briefly, $48 \mathrm{~h}$ after treatment, the MCF-7, MDA-MB-231 s and 4T1 cells were seeded into 96-well culture plates (BD Biosciences, Franklin Lakes, NJ, USA) and incubated overnight at $37{ }^{\circ} \mathrm{C}$ in $5 \% \mathrm{CO}_{2}$. Cell proliferation was assessed at $24,48,72,96$ and $120 \mathrm{~h}$ following addition of $0.5 \mathrm{mg} \mathrm{ml}^{-1}$ MTT (Sigma, USA) solution. After a 4-h incubation, the reaction was stopped by addition of $150 \mu \mathrm{l}$ DMSO (Sigma). After $10 \mathrm{~min}$ of agitation (100 rpm), the optical density (OD) at $490 \mathrm{~nm}$ was determined with microplate reader (BioTek). Each sample was tested with six replicates. All experiments were performed in biological triplicate.

\section{Wound migration assay}

The 4T1 cells were grown in six-well plates to a confluent monolayer and subsequently wounded with sterile pipette tips. The wounded monolayers were then incubated with lipopolysaccharide (LPS) (Sigma-Aldrich) and corresponding protein as indicated in Fig. $3 \mathrm{a}$ for $48 \mathrm{~h}$. The wound area was measured under microscope. The percentage of wound healing rate was estimated as follows: Wound healing rate $\%=[1-($ wound width at $48 \mathrm{~h} /$ wound width at $0 \mathrm{~h})] \times 100 \%$. $100 \times$ microscopic fields under microscope.

\section{Trans-well Matrigel invasion assay}

Tumor cell invasion was performed using Trans-well system (Millipore) with $8 \mu \mathrm{m}$-pore polycarbonate filter membrane. The upper chamber was covered with Matrigel (SigmaAldrich) and incubated at $4{ }^{\circ} \mathrm{C}$ overnight. The upper chamber was then seeded with $1 \times 10^{4} 4 \mathrm{~T} 1$ cells incubated with 
LPS and corresponding protein as indicated in Fig. 3b and inserted into the lower chamber containing complete medium. After incubation at $37{ }^{\circ} \mathrm{C}$ in $5 \% \mathrm{CO}_{2}$ for $24 \mathrm{~h}$, the cells on the interior of upper chamber were removed, and the polycarbonate membranes were stained with $0.1 \%$ crystal violet (BASO) for $10 \mathrm{~min}$. The number of migrating cells was counted in eight randomly selected $400 \times$ microscopic fields under microscope.

\section{Xenograft assays in nude mice}

To evaluate in vivo tumorigenesis, breast carcinoma xenografting mouse model was used. Male BALB/c mice weighing 18-22 g were obtained from the Wenzhou Medical University Animal Centre and prepared for tumor implantation. All experimental procedures involving animals were performed in accordance with animal protocols approved by the Institutional Animal Use and Care Committee of Wenzhou Medical University and performed according to the institutional ethical guidelines for animal experiment. The mice were randomly divided into four groups ( $n=8$ per group). 4T1-bearing mice were injected with $3 \times 10^{5} 4 \mathrm{~T} 1$ cells (i.v. through the tail vein) 3 days after being treated with $\mathrm{L} 6 \mathrm{H} 21$ (at $10 \mathrm{mg} \mathrm{kg}^{-1}$ or $5 \mathrm{mg} \mathrm{kg}^{-1}$ ) and saline by intragastric administration, respectively. To investigate the toxicity of L6H21, mice of the fourth group were only treated with $\mathrm{L} 6 \mathrm{H} 21$ at $10 \mathrm{mg} \mathrm{kg}^{-1}$ but without inoculation of $4 \mathrm{~T} 1$ cells. Each group of mice were then intragastrically administrated per day until death or day 60 . The survival curve was made to analyse the survival rate. When the mice were dead or at day 60 , the body weight data were collected.

\section{Statistical analysis}

For all data, statistical analysis was performed in SPSS 22.0 for Windows (SPSS Inc.). All data are shown as means \pm SDs. The statistical significance between groups was obtained by Student's $t$ test or one-way ANOVA test and the significance level was set at $p<0.05$.

\section{Results}

\section{MD2 expression in four kinds of cell lines}

In our experiment, we first used western blot assay to examined the expression of MD2 in normal breast cells (Hs 578Bst) and three kinds of breast carcinoma cell lines (MCF-7, MDA-MB-231 s and 4T1). These cells were lysed in logarithmic phase and the total protein was extracted. MD2 protein expression was then detected by western blot. As shown in (Fig. 1), MD2 expressed in each kinds of cell

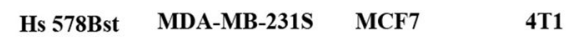

MD2

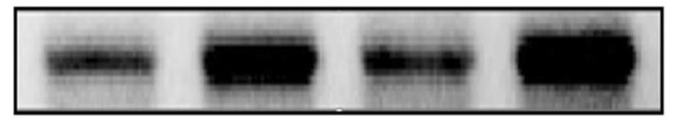

GAPDH

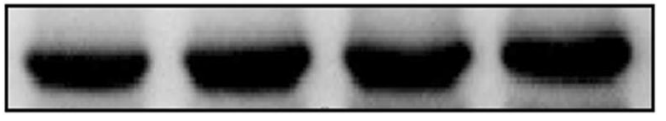

Fig. 1 MD2 is highly expressed in MDA-MB-231 s and 4T1 cells $(p<0.05$ compared to that expressed in Hs 578Bst cells and MCF-7 cells)

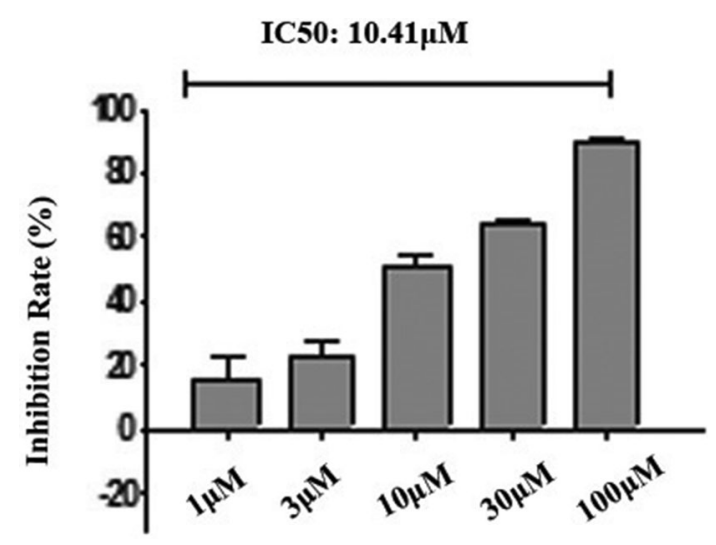

Fig. 2 MTT assay showed that L6H21 $(10 \mu \mathrm{M}, 30 \mu \mathrm{M}$ and $100 \mu \mathrm{M})$ significantly inhibited the proliferation of $4 \mathrm{~T} 1$ cells in dose-dependent manner

lines, of these, MD2 is highly expressed in highly malignant MDA-MB-231 s and 4T1 cells.

\section{L6H21 inhibition of breast cancer cells proliferation}

To explore the role of MD2 in breast cancer cells proliferation, we assessed the effects of inhibition of MD2 by L6H21 in 4T1 cells by MTT assay. As shown in (Fig. 2), L6H21 significantly inhibited the proliferation of $4 \mathrm{~T} 1$ cells in dosedependent manner $\left(\mathrm{IC}_{50}=10.41 \mu \mathrm{M}\right)$, which has a similar inhibition effect in each group by curcumin, respectively.

\section{L6H21 inhibition of breast cancer cells migration and invasion}

To study the effect of MD2 in cell migration, we adopted a scratch wound model in the presence of mitomycin C which inhibited proliferation. In these conditions, migration was significantly decreased in L6H21 group $(10 \mu \mathrm{M})$ and anti-MD2 group $(1 \mu \mathrm{g} / \mathrm{ml})$ as compared to the control group. Next, we performed trans-well invasion assay to investigate the effect of $\mathrm{L} 6 \mathrm{H} 21$ in $4 \mathrm{~T} 1$ cell invasion further. As shown in (Fig. 3), there were significant differences of 
Fig. 3 L6H21 inhibition of 4T1 cells migration and invasion. a L6H21 significantly inhibited the migration of $4 \mathrm{~T} 1$ cells, The images $(100 \times)$ were obtained by microscope. b L6H21 significantly inhibited the invasion of $4 \mathrm{~T} 1$ cells $(400 \times)$. In $5 \mu \mathrm{M}$ L6H21 group, there were a few migrating cells observed compared to that of control group. While in $10 \mu \mathrm{M}$ L6H21 group and in $1 \mu \mathrm{g} \mathrm{ml}^{-1}$ anti-MD2 group, there were almost no migrating cells observed

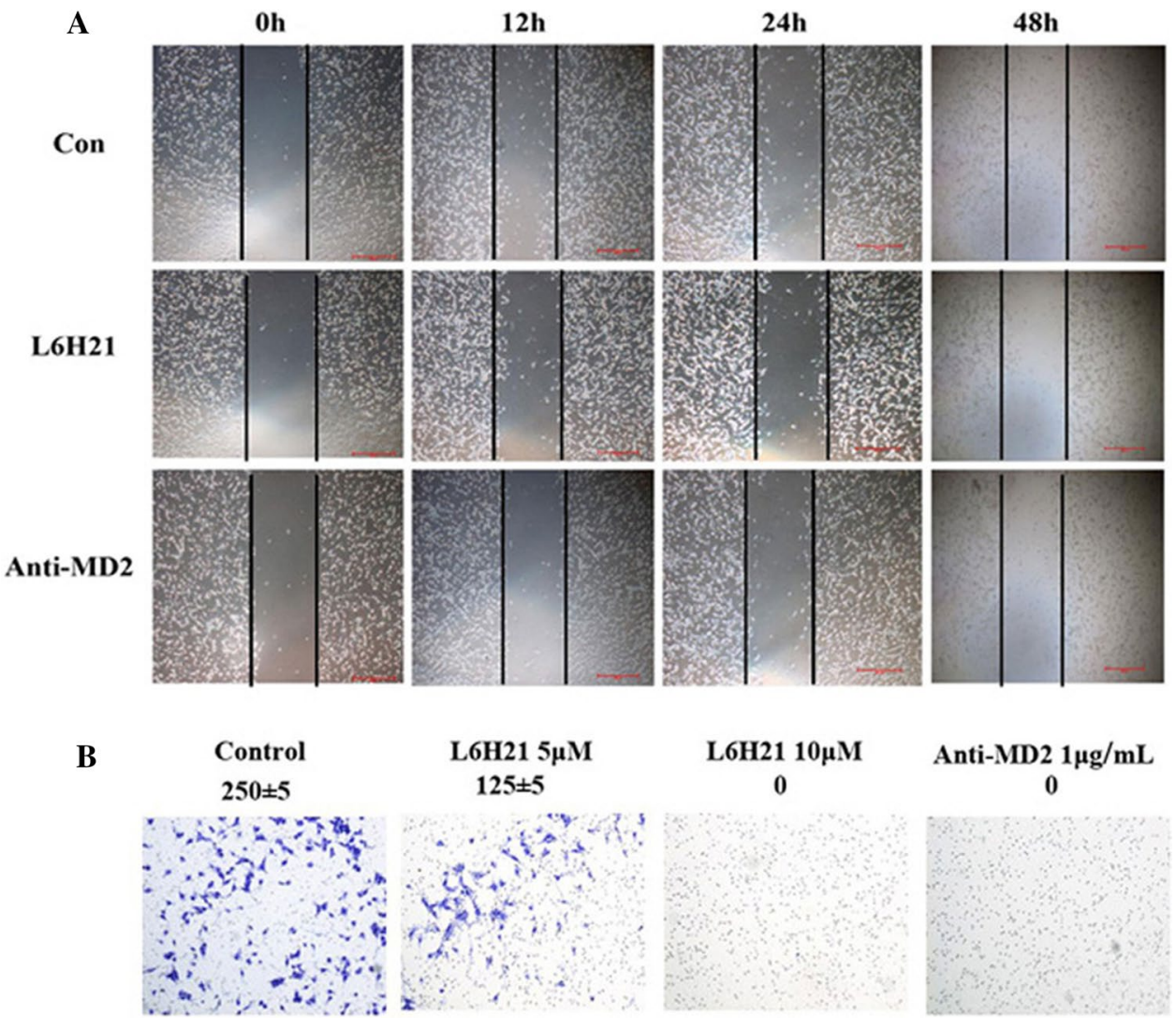

effect of invasion in control group, $5 \mu \mathrm{M}$ L6H21 group and $10 \mu \mathrm{M}$ L6H21 group. Moreover, there was almost no cell observed in $10 \mu \mathrm{M}$ L6H21 group as well as in $1 \mu \mathrm{g} \mathrm{ml}$ anti-MD2 group. These results indicate that $\mathrm{L} 6 \mathrm{H} 21$ has a significant inhibition of migration and invasion in 4T1 cell concentration-dependently.

\section{L6H21 suppresses tumor progression in the nude mice}

To further investigate the role of MD2 in tumor progression, we assessed the effects of inhibition of MD2 on the progression of xenograft tumors in vivo. Figure 4 shows

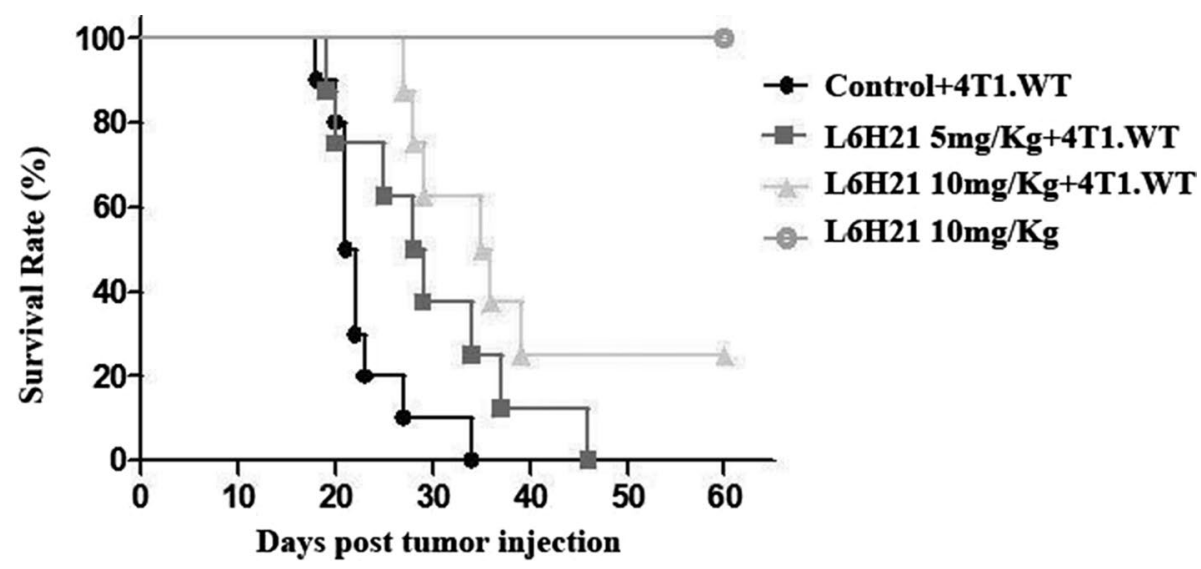

Fig. 4 L6H21 enhanced survival in nude mice. Male BALB/c mice were randomly divided into four groups ( $n=8$ per group). 4T1-bearing mice were injected with $3 \times 10^{5} 4 \mathrm{~T} 1$ cells (i.v. through the tail vein) 3 days after being treated with $\mathrm{L} 6 \mathrm{H} 21$ (at $10 \mathrm{mg} \mathrm{kg}^{-1}$ or $5 \mathrm{mg}$ $\mathrm{kg}^{-1}$ ) and saline by intragastric administration, respectively, and the mice of the fourth group were only treated with $\mathrm{L} 6 \mathrm{H} 21$ at $10 \mathrm{mg} \mathrm{kg}^{-1}$ but without inoculation of 4T1 cells. Each group of mice were then intragastrically administrated per day until death or day 60 . The survival curve was made to analyse the survival rate 
the survival rate of 4T1-bearing mice (treated with saline, $5 \mathrm{mg} \mathrm{kg}^{-1}$ and $10 \mathrm{mg} \mathrm{kg}^{-1} \mathrm{~L} 6 \mathrm{H} 21$, respectively) and toxic control mice (L6H21 treated but without inoculation of 4T1 cells). As expected, the $5 \mathrm{mg} \mathrm{kg}^{-1} \mathrm{~L} 6 \mathrm{H} 21$ - or $10 \mathrm{mg} \mathrm{kg}^{-1}$ L6H21-treated mice survived significantly $(p<0.05)$ longer than the saline-treated mice. The mean survival times of 4T1-bearing mice treated with saline, $5 \mathrm{mg} \mathrm{kg}^{-1}$ and $10 \mathrm{mg}$ $\mathrm{kg}^{-1} \mathrm{~L} 6 \mathrm{H} 21$ were $23.8 \pm 4.8,30.9 \pm 8.9,40.4 \pm 12.6$ days, respectively. In addition, after treated for 60 days, there were still two mice $(25 \%)$ survive in 4T1-bearing mice treated with $10 \mathrm{mg} \mathrm{kg}^{-1} \mathrm{~L} 6 \mathrm{H} 21$. Interestingly, we also observed that there was none of the mice died from the toxic of $10 \mathrm{mg} \mathrm{kg}^{-1}$ L6H21 in 60 days. Figure 5 shows the body weight data of four groups after treatment. On the one hand, there were no differences in body weights in $5 \mathrm{mg} \mathrm{kg}^{-1} \mathrm{~L} 6 \mathrm{H} 21$ - or $10 \mathrm{mg}$ $\mathrm{kg}^{-1}$ L6H21-treated 4T1-bearing mice as compared to the saline-treated 4T1-bearing mice $(p>0.05)$. But an upward trend of body weight could be seen as the concentration of L6H21 increase. On the other hand, the body weights of toxic control mice were significantly $(p<0.05)$ heavier than those of saline-treated 4T1-bearing mice. These data indicated that as an inhibitor of MD2, L6H21, could prolong survival efficiently with reliable security.

\section{Statistical analysis}

For all data, statistical analysis was performed in SPSS 22.0 for Windows (SPSS Inc.). All data are shown as means \pm SDs. The statistical significance between groups was obtained by Student's $t$ test or one-way ANOVA test and the significance level was set at $p<0.05$.

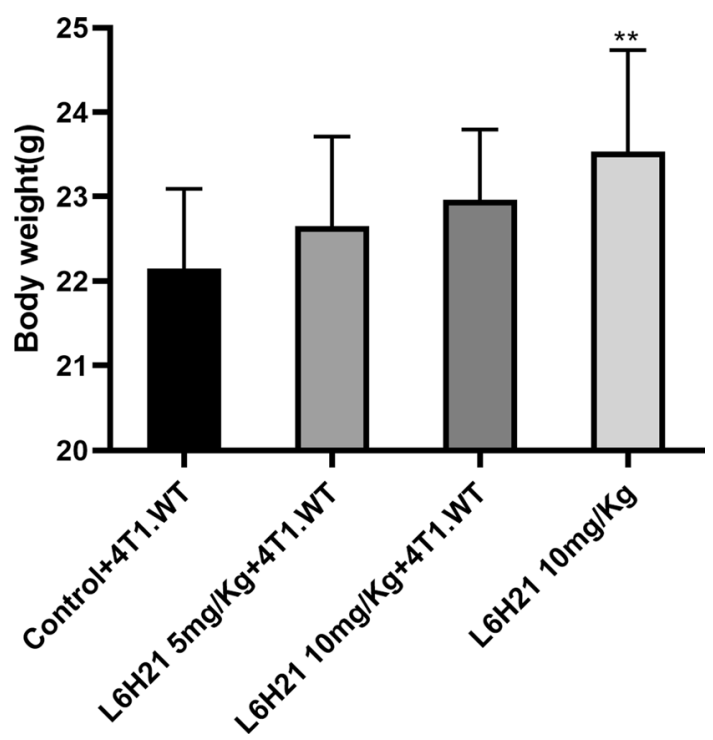

Fig. 5 Body weight data after treatment. The body weight data after treatment were collected when mice were dead or at day 60 . $* * p<0.05$ compared to the control group

\section{Discussion}

Diverse studies have shown that TLR4 is associated with tumor development and progression. In breast cancer, TLR4 activation has been linked to both cancer inhibition and growth [10,14-16]. Yang et al. reported that TLR4 expressed higher levels than any other TLRs and knockdown of TLR4 could actively inhibit proliferation and survival of human breast cancer cells MDA-MB-231. Functional analyses of ribonucleic acid interference (RNAi) against TLR4 revealed this successfully inhibited the growth and proliferation of MDA-MB-231 cells and resulted in a significant $(p<0.05)$ reduction of inflammatory cytokines [11]. In other work, $4 \mathrm{~T} 1$ cells challenged with lipopolysaccharide induced tumor growth and metastasis, by increasing angiogenesis, vascular permeability, and tumor invasion $[17,18]$. A total of 74 breast carcinomas were collected from patients to study the clinical relevance of TLRs in breast cancer. Tumors with high TLR4 expression, in mononuclear cells were found to have a higher probability of metastasis [19]. These studies suggest TLR4 involvement in breast cancer progression.

Although TLR4 plays an essential part in breast cancer progression, the role of its accessory protein, MD2, known for several years as an essential co-factor for TLR4 signaling, has not yet been clarified. One study highlighted that MD2 was overexpressed in highly invasive colorectal cancer cells (SW837), in poorly differentiated, moderately invasive colorectal cancer cells (HT-29), and in well-differentiated but non-invasive colorectal cancer cells (Caco2) [17]. Another study reported that serum amyloid A 3, a major component of acute inflammation, binds to MD2 and activates the MyD88-dependent TLR4/MD2 pathway and thus facilitates lung metastasis [21]. Therefore, MD2 could be related to the degree of differentiation, proliferation, and migration capacity of cancers. However, there was few research focus on the relationship between the MD2 expression and the progression of breast cancer.

In the first stage of our study, we detected the expression of MD2 in several cell lines. As expected, the western blot assay showed MD2 is highly expressed in MDAMB-231 s and 4T1 cells. Our preliminary results may probably indicate that MD2 is higher expressed in highly malignant cell lines than that in normal breast cells (Hs 578Bst) or MCF-7 cells $(p<0.05)$.

To investigate the role of MD2 in breast cancer progression, we next utilized L6H21, a new MD2 inhibitor, for down-regulating the expression of the MD2. And we performed experiments to observe the change of biological behavior of $4 \mathrm{~T} 1$ cells using L6H21. MTT assay was performed and the results showed that $\mathrm{L} 6 \mathrm{H} 21$ significantly inhibited the proliferation of 4T1 cells 
in dose-dependent manner, especially in $100 \mu \mathrm{M}$ group. Moreover, scratch wound model and trans-well invasion assay demonstrated that $\mathrm{L} 6 \mathrm{H} 21$ has a significant inhibition of migration and invasion in 4T1 cell. These results indicated that suppression of MD2 could effectively repress tumor cell proliferation, migration and invasion in vitro, in part consistent with the findings of Grondin et al., which was experimented with HT-29 cell [20]. To further determine whether MD2 regulates tumor progression in vivo, we used tumor xenografts by inoculating $4 \mathrm{~T} 1$ cells in L6H21-treated or saline-treated nude mice. And we provided evidence that MD2 suppression by L6H21 prolonged survival in nude mice with hypotoxicity.

\section{Conclusion}

In conclusion, our study indicates that $4 \mathrm{~T} 1$ cells treated with L6H21 show progression inhibition in vitro. Moreover, L6H21 can significantly prolong survival in vivo. To our knowledge, this is the first report to describe the significance of MD2 expression to breast cancer cells in vitro and in vivo. Although the precise molecular mechanisms behind the altered expression of MD2 in breast cancer remain poorly understood, our data suggest that MD2 may be a promising candidate as a potential therapeutic target for breast cancer intervention.

Acknowledgements We would like to thank the Natural Science Foundation of Zhejiang Province of China (LY19H160026), Zhejiang Provincial Health Department (2019KY454) and Wenzhou Bureau of Science and Technology grants (Y20170038, Y20170257), whose financial support has made this study possible.

Author contributions GLG and SRZ designed the study. SRZ and WDF conducted this experiment and RMM helped to design study model. WDF, SRZ, QDH, JWG, JYZ and KKL collected data. SRZ, WDF, RMM and QDH did the statistical analyses and prepared figures. SRZ, WDF and GLG reviewed the results and interpreted data. SRZ and WDF wrote the manuscript. All authors have made an intellectual contribution to the manuscript and approved the submission. WDF and SRZ are co-first authors.

Funding This work was funded by the Natural Science Foundation of Zhejiang Province of China (LY19H160026), Zhejiang Provincial Health Department (2019KY454) and Wenzhou Bureau of Science and Technology grants (Y20170038, Y20170257).

Availability of data and material The dataset used during the present study is available from the corresponding author upon a reasonable request.

Code availability Not applicable.

\section{Declarations}

Conflict of interest The authors declare that they have no conflict of interest.

Ethics approval The present study was approved by the Medical Ethical Committee of the First Affiliated Hospital of Wenzhou Medical University and conformed to the provisions of the Declaration of Helsinki in 1995 (as revised in Edinburgh 2000). Protocols number approved by the Institutional Animal Use and Care Committee of Wenzhou Medical University: wydw2019-0487.

Informed consent This research did not involve human participants performed by any of the authors. For this type of study, formal consent is not required.

Consent to participate This research did not involve human participants performed by any of the authors. For this type of study, formal consent is not required.

Consent for publication This article has been read and approved in the present form for submission by all authors.

Open Access This article is licensed under a Creative Commons Attribution 4.0 International License, which permits use, sharing, adaptation, distribution and reproduction in any medium or format, as long as you give appropriate credit to the original author(s) and the source, provide a link to the Creative Commons licence, and indicate if changes were made. The images or other third party material in this article are included in the article's Creative Commons licence, unless indicated otherwise in a credit line to the material. If material is not included in the article's Creative Commons licence and your intended use is not permitted by statutory regulation or exceeds the permitted use, you will need to obtain permission directly from the copyright holder. To view a copy of this licence, visit http://creativecommons.org/licenses/by/4.0/.

\section{References}

1. Li N, Deng Y, Zhou L, et al. Global burden of breast cancer and attributable risk factors in 195 countries and territories, from 1990 to 2017: results from the Global Burden of Disease Study 2017. J Hematol Oncol. 2019;12:140.

2. Fidler IJ. The pathogenesis of cancer metastasis: the 'seed and soil' hypothesis revisited. Nat Rev Cancer. 2003;3:453-8.

3. Lucas K, Maes M. Role of the toll like receptor (TLR) radical cycle in chronic inflammation: possible treatments targeting the TLR4 pathway. Mol Neurobiol. 2013;48:190-204.

4. Korneev KV, Atretkhany K-SN, Drutskaya MS, et al. TLR-signaling and proinflammatory cytokines as drivers of tumorigenesis. Cytokine. 2017;89:127-35.

5. Khademalhosseini M, Arababadi MK. Toll-like receptor 4 and breast cancer: an updated systematic review. Breast Cancer. 2019;26:265-71.

6. Bhatelia K, Singh K, Singh R. TLRs: linking inflammation and breast cancer. Cell Signal. 2014;26:2350-7.

7. Zhang YB, He FL, Fang M, et al. Increased expression of Tolllike receptors 4 and 9 in human lung cancer. Mol Biol Rep. 2009;36:1475-81.

8. Kelly MG, Alvero AB, Chen R, et al. TLR-4 signalling promotes tumor growth and paclitaxel chemoresistance in ovarian cancer. Cancer Res. 2006;66:3859-68. 
9. Zhou Y-H, Liao S-J, Li D, et al. TLR4 ligand/ $\mathrm{H}_{2} \mathrm{O}_{2}$ enhances TGF- $\beta 1$ signaling to induce metastatic potential of non-invasive breast cancer cells by activating non-Smad pathways. PLoS ONE. 2013;8:e65906.

10. Lin Y-S, Huang L-D, Lin C-H, et al. In vitro and in vivo anticancer activity of a synthetic glycolipid as Toll-like receptor 4 (TLR4) activator. J Biol Chem. 2011;286:43782-92.

11. Yang H, Zhou H, Feng P, et al. Reduced expression of Tolllike receptor 4 inhibits human breast cancer cells proliferation and inflammatory cytokines secretion. J Exp Clin Cancer Res. 2010;29:92.

12. Duan G, Zhu J, Xu J, et al. Targeting myeloid differentiation 2 for treatment of sepsis. Front Biosci (Landmark Ed). 2014;19:904-15.

13. Wang Yi, Shan X, Chen G, et al. MD-2 as the target of a novel small molecule, L6H21, in the attenuation of LPS-induced inflammatory response and sepsis. Br J Pharmacol. 2015;172:4391-405.

14. Azuma I, Seya T. Development of immunoadjuvants for immunotherapy of cancer. Int Immunopharmacol. 2001;1:1249-59.

15. Okamoto M, Furuichi S, Nishioka Y, et al. Expression of toll-like receptor 4 on dendritic cells is significant for anticancer effect of dendritic cell-based immunotherapy in combination with an active component of OK-432, a streptococcal preparation. Cancer Res. 2004;64:5461-70.

16. Okamoto M, Sato M. Toll-like receptor signaling in anti-cancer immunity. J Med Invest. 2003;50:9-24.
17. Harmey JH, Bucana CD, Lu W, et al. Lipopolysaccharide-induced metastatic growth is associated with increased angiogenesis, vascular permeability and tumor cell invasion. Int $\mathrm{J}$ Cancer. 2002;101:415-22.

18. Ahmed A, Wang JH, Redmond HP. Silencing of TLR4 increases tumor progression and lung metastasis in a murine model of breast cancer. Ann Surg Oncol. 2013;20(Suppl 3):S389-96.

19. González-Reyes S, Marín L, González L, et al. Study of TLR3, TLR4 and TLR9 in breast carcinomas and their association with metastasis. BMC Cancer. 2010;10:665.

20. Grondin V, Seksik P, Dumont S, et al. Regulation of colon cancer cell proliferation and migration by MD-2 activity. Innate Immun. 2011;17:414-22.

21. Deguchi A, Tomita T, Omori T, et al. Serum amyloid A3 binds MD-2 to activate $\mathrm{p} 38$ and NF- $\kappa$ B pathways in a MyD88-dependent manner. J Immunol. 2013;191:1856-64.

Publisher's Note Springer Nature remains neutral with regard to jurisdictional claims in published maps and institutional affiliations. 